\title{
Novel Bionic Design Method for Skeleton Structures Based on Load Path Analysis
}

\author{
Zhaohua Wang ${ }^{1}{ }^{(}$, Nan $\mathrm{Wu}^{2}{ }^{2}$, , Qingguo Wang ${ }^{3}$, Yongxin $\mathrm{Li}^{1}{ }^{1}$, Quanwei Yang ${ }^{1}$ and \\ Fenghe $\mathrm{Wu}^{1}$,* \\ 1 School of Mechanical Engineering, Yanshan University, Qinhuangdao 066004, China; \\ wangzh@stumail.ysu.edu.cn (Z.W.); liyongxin@ysu.edu.cn (Y.L.); qw_yangz@163.com (Q.Y.) \\ 2 Department of Mechanical Engineering, University of Manitoba, Winnipeg, MB R3T 5V6, Canada; \\ Nan.Wu@umanitoba.ca \\ 3 Buhler Versatile Inc., Winnipeg, MB R3T 1T2, Canada; qwang@versatile-ag.com \\ * Correspondence: risingwu@ysu.edu.cn; Tel.: +86-130-3188-3820
}

Received: 29 October 2020; Accepted: 19 November 2020; Published: 20 November 2020

\begin{abstract}
Biological structures have excellent mechanical performances including lightweight, high stiffness, etc. However, these are difficult to apply directly to some given complex structures, such as automobile frame, control arm, etc. In this study, a novel bionic design method for skeleton structures with complex features is proposed by the bio-inspired idea of "main-branch and sub-branch". The envelope model of a given part is established by analyzing the structural functions and working conditions, and the load path is extracted by the load-transferred law as the structural main-branch. Then, the selection criterion of bionic prototype is established from three aspects: load similarity, structural similarity and manufacturability. The cross-sections with high similarities are selected as the structural sub-branch. Finally, the multi-objective size optimization is carried out and a new model is established. The bionic design of a control arm is carried out by the method: structural main-branch is obtained by the load path analysis and structural sub-branch is occupied by the fish-bone structure. The design result shows that the structural stiffness is increased by $62.3 \%$, while the weight is reduced by $24.75 \%$. The method can also be used for other fields including automobile, aerospace and civil engineering.
\end{abstract}

Keywords: bionic design; lightweight; load path; skeleton structures; control arm

\section{Introduction}

The skeleton structures have been widely used in vehicles, aerospace, aviation and civil engineering because of their universality and foundation. As the lightweight is becoming an inevitable trend in these fields, the skeleton structures can usually be designed as thin-walled structures, including thin plates, shells and slender members [1,2]. Biological structures have excellent mechanical performance after hundreds of millions of years of evolution, which provided structural engineers with a lot of creative ideas for addressing the lightweight demands, such as bamboo, honeycomb, waterlily, bone and so on $[3,4]$. If the biological characteristics are transferred to the engineering structures in the design stage, a lot of creative ideas for structural engineers will be provided to solve the lightweight demand. Therefore, structural bionic lightweight design has become a research hotspot $[5,6]$.

In recent years, many achievements applying biological structures to engineering equipment to achieve lightweight have been carried out [7]. Wang et al. [8] designed an anti-collision beam vehicle with functional gradient features according to the human tibia porous gradient with good bearing and impact resistance, which effectively improved the crashworthiness and energy absorption performance of the anti-collision beam. Zhao et al. [9] applied the growth law of the waterlily 
and cactus to the cross beam of gantry machining center by analyzing the distribution law of the main and sub branch veins. The advantages of the bionic model were verified by simulation and experiment. Kim et al. [10] applied sandwich structure to the layout of ribs for micro-EDM machine tool, which improved structural bearing performance. These achievements are based on the full understanding of the functions and working conditions of parts and then the bionic prototypes with high similarity in structure are selected by human intuition and transferred to engineering structures [11]. However, for those complex structures, especially the skeleton structures, such as automobile frame, steering knuckle, aircraft landing gear, control arm etc., it is difficult to find a bionic prototype with high similarity in structure. For example, the car frame requires high collision resistance [12], but it is a typical multi-branch skeleton structure with multiple load paths, and it is difficult to find a bionic prototype with similar features.

In order to expand the application of bionic design, some scholars [13-15] took natural branching systems as bionic objects to study the optimum morphology and growth mechanism. A bio-adaptive growth algorithm is proposed and the growth law of stiffeners is obtained by numerical calculation, which skillfully avoids the selection of bionic prototype. However, it is only suitable for the layout optimization of stiffeners in the box structures. Some scholars [16-18] combined the theory of the solution of inventive problems (TRIZ) with bionic design and used the conflict matrix to guide the selection of bionic prototype, which improves the efficiency to a certain extent. But it is still limited to parts with similar structures or functions to bionic prototypes. In addition, some scholars try to guide the bionic design by topology optimization. Emmelmann et al. [19] used the topology optimization to get the load transfer skeleton of the aircraft bracket and then applied the porous structure to the load transfer skeleton, the lightweight aircraft bracket is obtained by additive manufacturing (AM). Maier et al. [20] applied the porous structure of biological microstructure to the bionic design and introduced the topology optimization to solve the boundary problem of the bionic model. Although topology optimization can reduce human subjectivity, its calculation process is relatively complex and the obtained load transfer skeleton often has many branches and holes [21,22], which makes selection of bionic prototypes more difficult. In addition, the combination of topology optimization and porous structure of biological micro-structure makes the manufacturing process of these hollow structures very poor. It is difficult to manufacture directly by traditional mechanical processing, which restricts the engineering application of bionic design. The development of AM promotes the manufacturability of this kind of difficult-to-process structures to a certain extent $[23,24]$. However, due to its long processing time, high material cost and process defects, the performance of parts is lower than that of traditional manufacturing technology, which leads to some difficulties in engineering application. Therefore, in the process of bionic design, if we can fully consider the manufacturing process and make the model adopt the traditional manufacturing method as far as possible, it will greatly improve the engineering application.

Based on the above background, it is known that new bionic design methods are needed to improve the efficiency and effectiveness of bionic design, especially for complex structures such as vehicle components. The difficulties in selecting bionic prototypes for the whole structure could be avoid if the main load-transferred skeleton is extracted as the bionic main-branch and the bionic prototype is then selected for each sub-branch based on the specific load-transferred features. In this way, the efficiency of bionic design would be significantly improved.

In this study, a novel bionic design method based on load path analysis is proposed by the bio-inspired design idea of main-branch and sub-branch to address the above-mentioned problems for the skeleton structures. The sections are organized as follows: the bionic design method is elaborated on in detail in Section 2. Taking the control arm of automobile suspension system as an example, the bionic design is carried out in Section 3. Section 4 verifies the effectiveness of bionic design followed by the conclusion in Section 5 . 


\section{Methodology}

The bionic design method proposed in this paper is shown in Figure 1, which is divided into the following five steps.

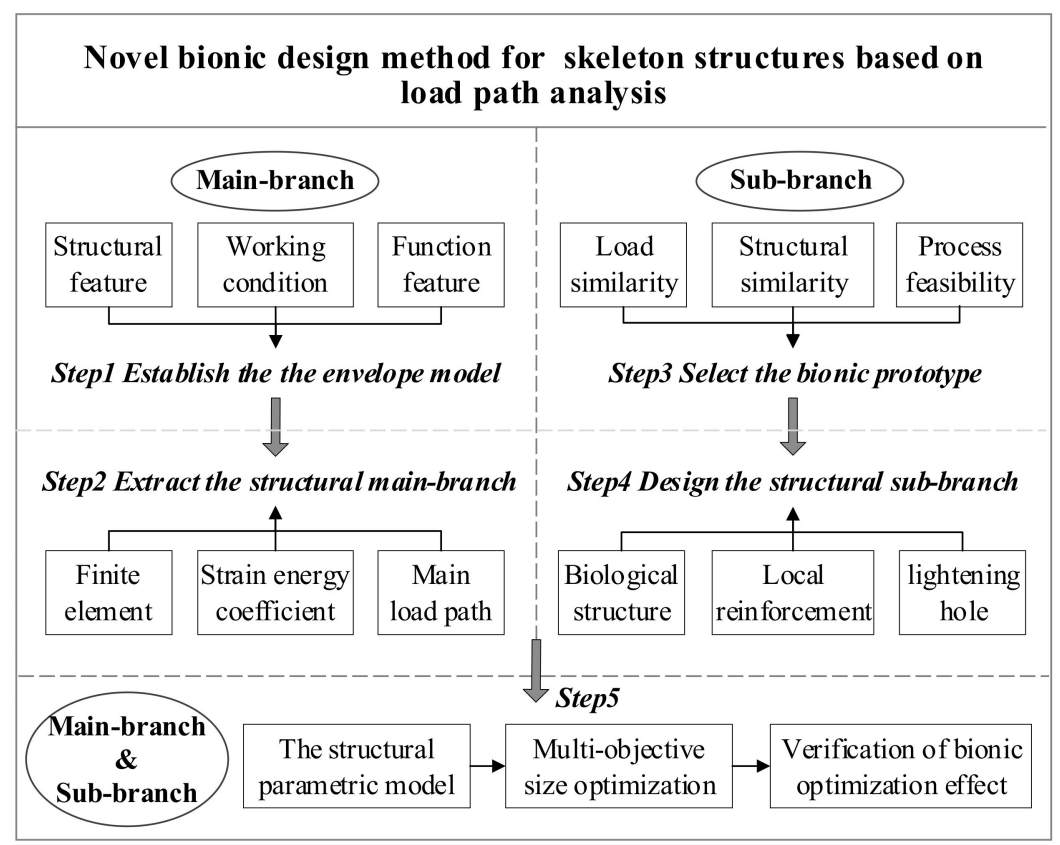

Figure 1. Bionic design method based on the idea of main-branch and sub-branch.

(1) The structure, function and working condition of a given part is analyzed and the corresponding envelope model is established.

(2) The main load path of the envelope model is extracted by the load path analysis as the main-branch of the new structure.

(3) According to the load-bearing characteristics of the main-branch, the bionic prototype is selected from three aspects: load similarity, structural similarity and manufacturability, which transform the whole bionics into local bionics.

(4) The cross-sectional characteristics of the bionic prototype are analyzed and the bionic sub-branch is designed.

(5) Based on the idea of main-branch and sub-branch, the bionic parametric model is established and the final bionic lightweight model is obtained by the multi-objective size optimization. Finally, the mechanical performances of the bionic model are verified by finite element analysis (FEA).

\subsection{Establish the Envelope Model}

It is necessary to abandon the structural features designed by traditional design methods that may interfere with the extraction of load path in next step. This method firstly analyzes the working conditions and structural characteristics of research objects, fills the internal holes and then establishes the envelope model. Taking a cantilever bracket as an example, the process of establishing the envelope model is introduced in detail as shown in Figure 2, and the basic criteria are as follows:

(1) The working conditions of the parts are analyzed to ensure that the constrained and loaded areas remain unchanged. As shown in Figure $2 b$, the loaded and constrained areas of the bracket represented by red lines remain unchanged.

(2) The internal structure and function of the parts are analyzed and the internal holes that do not affect the regular work are filled. As shown in Figure 2c, the two lightening holes removed in the middle of the bracket are denoted by green dotted lines. 
(3) The outer boundary of the parts is analyzed and the complex boundary is filled into the regular boundary. As shown in blue lines in Figure 2d, the regular outline of the parts is finally obtained.

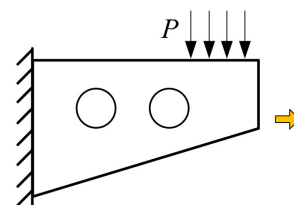

(a)

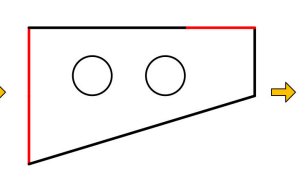

(b)

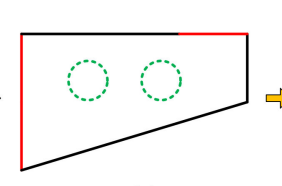

(c)

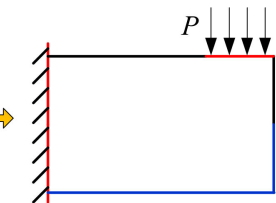

(d)

Figure 2. Establishing the envelope model. (a) a cantilever bracket, (b) the contrained and loaded areas remain unchanged, (c) the lightening holes are removed, $(\mathbf{d})$ the regular outline of the bracket.

\subsection{Extract the Structural Main-Branch}

In this paper, the main-branch of the new structure is extracted by the load path theory. In general, it can give full play to the load-bearing performance and achieve lightweight at the source if the materials are arranged along the main load path in the design stage. In recent years, more and more researchers are interested in the load path visualization [25-28]. Among them, Takahashi et al. [29,30] proposed a load path method, which holds that the load always transfers along the channel with the largest relative stiffness in the structure according to a given working condition. Wang et al. [31,32] applied this method to the structural design of automobile body and luggage rack, the main load path is visualized and structural load-bearing performance is improved. Compared with other methods of load path, this method has clear theoretical basis, physical significance and abundant application cases. Therefore, the main load path is extracted by this method as the main-branch of the new structure.

The calculation process of the load path visualization based on the relative stiffness [33] is shown in Figure 3. Firstly, the finite element model (FEM) of the envelope structure is established and the strain energy is calculated. Secondly, one node is fixed in turn and the structural strain energy is calculated again. Then, the stiffness between each node and the loading point is calculated according to the strain energy coefficient. Finally, the main load path, i.e., the load transfer skeleton, is fitted according to the strain energy coefficient cloud chart. The detailed calculation process is as follows:

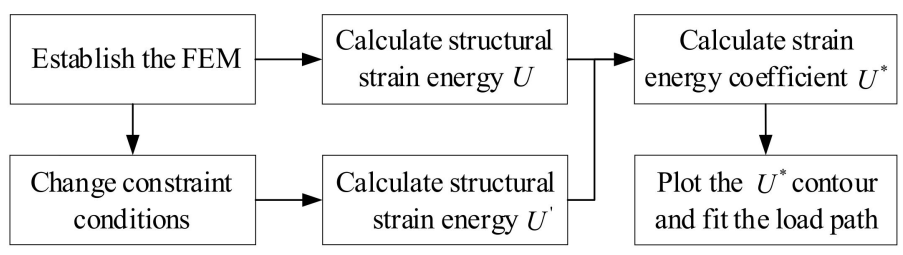

Figure 3. Flow chart of the load path calculation.

The elastic structure is simplified to the model shown in Figure 4a, which includes loading point A, supporting point $B$ and an arbitrary point $C$. It is assumed that the three points are connected by springs which represent the relative stiffness between any two points of the system.

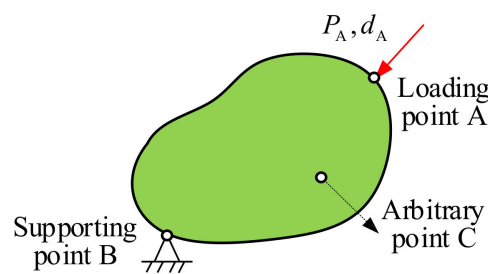

(a)

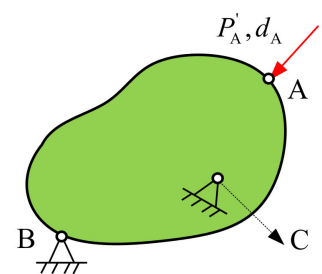

(b)

Figure 4. Simplified model of an elastic structure: (a) original system; (b) constrained system. 
According to the concept of relative stiffness, which is the coupling stiffness between any two points of the structure, the load-displacement relationship among points $\mathrm{A}, \mathrm{B}$ and $\mathrm{C}$ can be expressed as:

$$
\left\{\begin{array}{c}
P_{\mathrm{A}} \\
P_{\mathrm{B}} \\
P_{\mathrm{C}}
\end{array}\right\}=\left[\begin{array}{c}
K_{\mathrm{AA}} K_{\mathrm{AB}} K_{\mathrm{AC}} \\
K_{\mathrm{BA}} K_{\mathrm{BB}} K_{\mathrm{BC}} \\
K_{\mathrm{CA}} K_{\mathrm{CB}} K_{\mathrm{CC}}
\end{array}\right]\left\{\begin{array}{c}
d_{\mathrm{A}} \\
d_{\mathrm{B}} \\
d_{\mathrm{C}}
\end{array}\right\}
$$

where $k_{i j(i, j=\mathrm{A}, \mathrm{B}, \mathrm{C})}$ is the internal stiffness tensor, $P_{i(i=\mathrm{A}, \mathrm{B}, \mathrm{C})}$ and $d_{i(i=\mathrm{A}, \mathrm{B}, \mathrm{C})}$ represent the load and the displacement vectors, respectively.

Applying load $P_{\mathrm{A}}$ at point A produces displacement $d_{\mathrm{A}}$, the total strain energy $U$ of the model due to the external load is calculated according to the strain energy formula:

$$
U=\frac{1}{2} P_{\mathrm{A}} d_{\mathrm{A}}
$$

Since point $\mathrm{B}$ is fixed $\left(d_{\mathrm{B}}=0\right), P_{\mathrm{A}}$ is calculated by Equation (1),

$$
P_{\mathrm{A}}=K_{\mathrm{AA}} d_{\mathrm{A}}+K_{\mathrm{AC}} d_{\mathrm{C}}
$$

So the total strain energy $U$ is:

$$
U=\frac{1}{2} P_{\mathrm{A}} d_{\mathrm{A}}=\frac{1}{2}\left(K_{\mathrm{AA}} d_{\mathrm{A}}+K_{\mathrm{AC}} d_{\mathrm{C}}\right) d_{\mathrm{A}}
$$

In Figure $4 \mathrm{~b}$, any point $\mathrm{C}$ in the structure is fixed and the same displacement $d_{\mathrm{A}}$ is produced by applying load $P_{\mathrm{A}}^{\prime}$ at point $\mathrm{A}$. The total strain energy $U^{\prime}$ is:

$$
U^{\prime}=\frac{1}{2} P_{\mathrm{A}}^{\prime} d_{\mathrm{A}}=\frac{1}{2}\left(K_{\mathrm{AA}} d_{\mathrm{A}}\right) d_{\mathrm{A}}
$$

In order to express the stiffness relationship between an arbitrary point and the loading point in the structure, the dimensionless coefficient $U^{*}$ is defined as:

$$
U^{*}=\frac{U^{\prime}-U}{U^{\prime}}=\left(1-\frac{2 U}{\left(K_{\mathrm{AC}} d_{\mathrm{C}}\right) \cdot d_{\mathrm{A}}}\right)^{-1}
$$

Equation (6) indicates that the $U^{*}$ only varies with $K_{\mathrm{AC}}$ and $U^{*}$ can characterize the internal stiffness between an arbitrary point and loading point of the structure.

In an elastic structure, the applied load is mainly transferred through the locations having the strongest coupling stiffness with the loading point. Therefore, connecting nodes with the same stiffness to form a cluster of stiffness contours, the line with the highest $U^{*}$ values which is the ridge line of the $U^{*}$ distribution is the main load path. So, if a vector $\lambda$ is introduced as:

$$
\lambda=-\operatorname{grad} U^{*}
$$

Then, the successively traced line along the smallest $\lambda$ value is the load path. In a simple case like the structure shown in Figure 5, the main load path is fitted by the $U^{*}$ distribution.

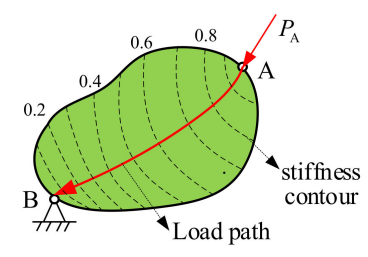

Figure 5. The main load path for a sample structure. 


\subsection{Select the Bionic Prototype of the Main-Branch}

The structure is still very bulky if the material is laid directly on the structural main load path obtained in Section 2.2. Therefore, it is necessary to establish a lightweight and efficient cross-section on the main load path. Under the influence of heredity and evolution, organisms have gradually formed their own unique cross sections, which provide a great deal of inspiration for the design of structural sub-branch. For example, the plant branches are slender and long, but the fractal structure of their veins can disperse the external loads, so that they can withstand larger bending moments in complex external environment [34].

In this paper, the loads on a given structure are equivalent to the main-branch of bionic structure, and the bionic prototype of each main-branch is selected respectively from three aspects: load similarity, structural similarity and manufacturability, which changes the principle of selecting bionic prototype from the similarity of whole structure or function to local. Besides, manufacturability needs to be considered as shown in Figure 6. The specific principles for selecting bionic prototypes of main-branch are as follows:

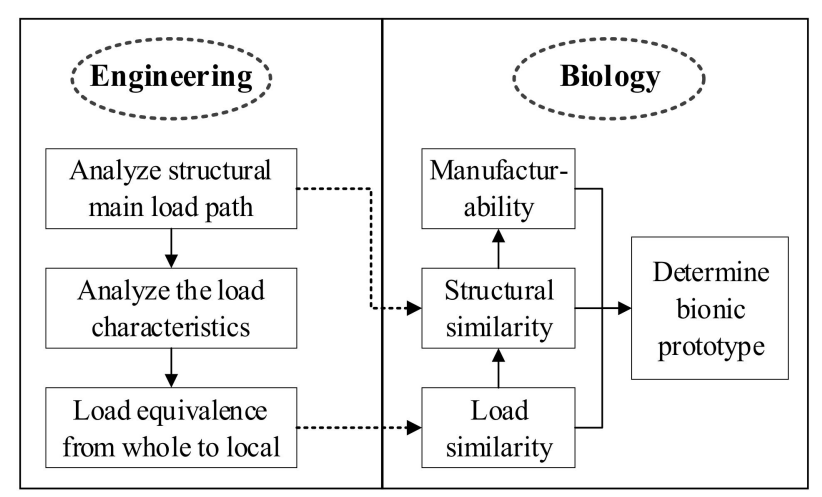

Figure 6. Selection of bionic prototype.

\subsubsection{Load Similarity}

It is the key of the bionic design to find a bionic prototype with similar load to the structural main-branch. The main-branch of the bionic structure has been established in Section 2.2, which can theoretically guarantee the mechanical performances. Therefore, the bionic prototype, which is similar to the load mode of the local main-branch, can be selected. For example, the bone mainly bears tension and pressure [35] and the plant stem mainly bears bending load [36].

\subsubsection{Structural Similarity}

Biological structures evolve by continuous self-optimization. The efficiency of bionic design can be improved by choosing bionic prototype similar to the main-branch in structure. Therefore, it is necessary to apply the cross-section of bionic prototype similar to the main-branch to the bionic sub-branch when choosing bionic prototype.

\subsubsection{Manufacturability}

In nature, there are many organisms with superior mechanical properties that are suitable for bionic design of hollow structures, such as bamboo, bone, etc. However, these structures are difficult to manufacture by traditional technology. Even if it can be produced by the AM, the final products still cannot be used in large quantities in engineering due to its process defects. Therefore, in the process of selecting bionic prototypes, we try not to consider this kind of bionic prototype with a hollow structure. 


\subsection{Design the Structural Sub-Branch}

After the bionic prototype is determined, the features of cross-section will be applied to bionic sub-branch to realize lightweight. The specific design principles are as follows:

(1) Analyze the growth law of biological structure. The section characteristics of the bionic prototype are analyzed and mapped to the main-branch as the basic topology and shape.

(2) Local reinforcement. Structural reinforcement design is carried out in areas with large loads, such as the application of reinforcement bars.

(3) Add lightening holes. The lightening holes are added in the areas with small load to achieve further lightweight.

(4) Manufacturability. In the process of bionic design, the structure should not be too complex, and the structural shape should not be too sharp or abrupt to avoid stress concentration.

\subsection{Multi-Objective Size Optimization}

After the bionic design of main-branch and sub-branch, the bionic model is established and the structural lightweight is realized to a great extent. However, bionic design only provides lightweight and efficient structural features in the conceptual design stage and cannot provide detailed size parameters. Size optimization can optimize local parameters based on existing topological structure and macro-size and then achieve secondary lightweight. Therefore, size optimization is carried out in this paper.

The starting point of this paper is to achieve structural lightweight by bionic design. So, the minimum weight, displacement and stress are set as optimization goals. In order to avoid the problem of low calculation efficiency caused by too many dimensions, the size parameters which have great influence on the structural weight, stiffness and strength are selected through the sensitivity analysis [37]. So, the mass sensitivity, displacement sensitivity and stress sensitivity are calculated by the direct derivation method in the Equation (8).

$$
\left\{\alpha_{M}, \alpha_{d}, \alpha_{\sigma}\right\}=\left\{\frac{\partial M}{\partial x_{i}}, \frac{\partial d}{\partial x_{i}}, \frac{\partial \sigma}{\partial x_{i}}\right\}
$$

where the $M, d, \sigma$ are the mass, displacement and stress, respectively. $\alpha_{M}, \alpha_{d}, \alpha_{\sigma}$ are the mass sensitivity, displacement sensitivity and stress sensitivity. $x_{i}$ is the vector of design variables.

Besides, the size should be set in a certain range as the constraint condition and the mathematical model of multi-objective size optimization is established as shown in Equation (9).

$$
\left\{\begin{array}{l}
\text { find } x_{i} \quad(i=1,2, \ldots, n) \\
\min f(x)=\min \left[M\left(x_{i}\right), d_{j}\left(x_{i}\right), \sigma_{j}\left(x_{i}\right)\right]^{T} \quad(j=1,2, \ldots, m) \\
\text { s.t. } x_{i} \in\left[r_{i 1}, r_{i 2}\right]
\end{array}\right.
$$

where $n$ denotes the number needed to be optimized and $m$ denotes the number of working conditions. $x_{i}$ is the design variable, $r_{i 1}$ and $r_{i 2}$ represent the upper and lower limits of $x_{i} . M\left(x_{i}\right)$ is the structural overall mass, while $d_{j}\left(x_{i}\right)$ and $\sigma_{j}\left(x_{i}\right)$ represent the maximum displacement and stress under the $j$-th working condition.

\section{Case Study}

The control arm is a component for transferring and guiding loads in the vehicle suspension system. Its performance directly affects the stability and reliability of the vehicle. In order to ensure stable driving and sensitive steering, the control arm is required to be small enough in weight on the basis of satisfying its stiffness and strength. In this paper, a control arm is taken as a case study to validate the proposed method. The installation position of the control arm in the automobile is shown in Figure 7 [38] and the structure and boundary conditions are shown in Figure 8. 


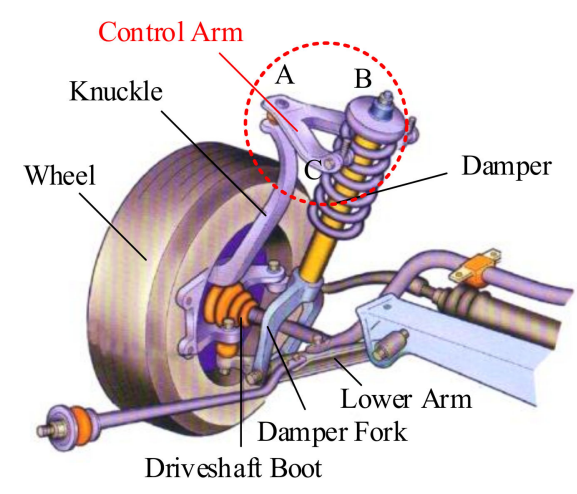

(a)

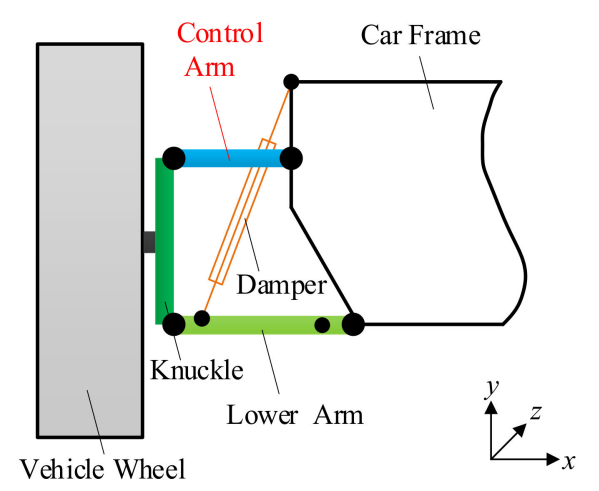

(b)

Figure 7. Suspension system diagram. (a) The schematic diagram of suspension system [38]; (b) the front view of the suspension system.

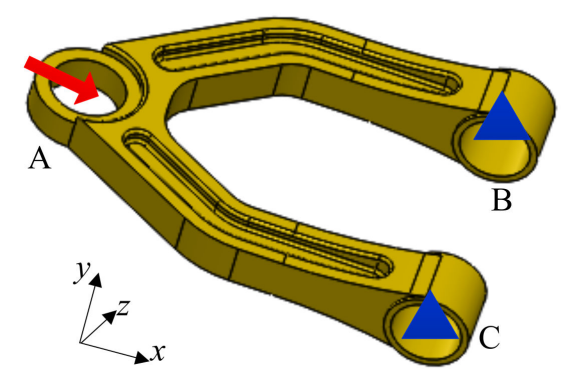

Figure 8. The control arm and boundary conditions.

The material is aluminum alloy A356.1 and material properties are given in Table 1. The two cylindrical holes (B and C) on the right side are directly connected with the vehicle frame, which can be equivalent to fixed constraints, while the cylindrical hole $\mathrm{A}$ on the left side is connected with the steering knuckle, which can be equivalent to pin load. During the driving process, the control arm has several different working conditions, including hitting the side of road (Condition 1), vehicle side slip and brake (Condition 2), left or right steering (Condition 3) and the fatigue life. Among these conditions, the Condition 1 is the most extreme, while the force of Condition 2 and Condition 3 is far less than Condition 1. Following the engineer's experience, only the loading condition with the positive load $3062.36 \mathrm{~N}$ in $x$ direction is considered in the process of bionic design. This case aims to validate the proposed method, so the other working conditions will not be considered.

Table 1. Material properties.

\begin{tabular}{ccccc}
\hline Material & Elastic Modulus (GPa) & Poisson's Ratio & Density (kg.m ${ }^{-3}$ ) & Yield Strength (MPa) \\
\hline A356.1 & 72.4 & 0.33 & 2680 & 220 \\
\hline
\end{tabular}

\subsection{Envelope Model of the Control Arm}

As the control arm in Figure 8 is designed by engineering experience, its topology structure is not optimal. In order to eliminate the disturbance of the original topology structure to the bionic design, the envelope model is established at first.

Three cylindrical holes (A, B and C) are the loaded and constrained areas, which must be retained. Then, the interference of the original topological structure is removed by changing the 
optimizable region to a regular rectangle. In addition, in the suspension system, the active space of the shock absorber should be set aside between the two constrained cylindrical holes (B and C). Finally, the envelope model of the control arm is established, as shown in Figure 9.

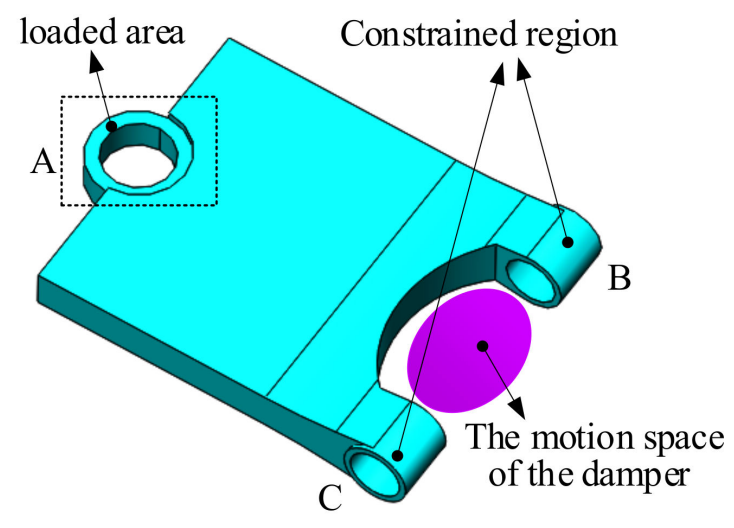

Figure 9. The envelope model of the control arm.

\subsection{Main-Branch of the Control Arm}

The main load transfer skeleton of the envelope model is calculated by the load path theory in Figure 3, which is used as the main-branch of control arm. The result is shown in Figure 10a and two main load paths represented by black lines are fitted according to the contour of strain energy coefficient $U^{*}$. The materials are arranged along two main load paths and the main-branch of the control arm is established as shown in Figure 10b. If two main-branches are designed as circular or other irregular sections, it will be very difficult to manufacture by traditional processing methods. Therefore, the bionic main-branch is designed as a rectangular section.

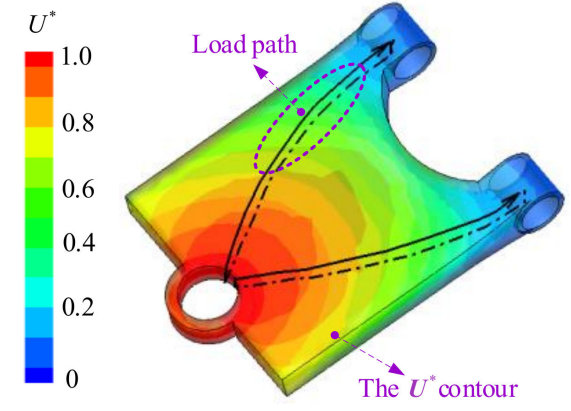

(a)

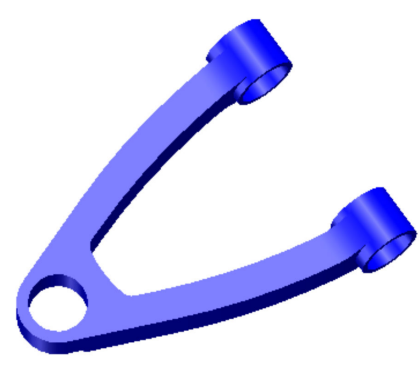

(b)

Figure 10. The main-branch of the control arm: (a) load path visualization; (b) two main-branches.

\subsection{Bionic Prototype of the Main-Branch}

From Figure 8 , it can be seen that the control arm mainly bears axial pressure along the $x$ direction in the extreme working condition, and it also have left and right bending loads along the $z$ direction while the car is in driving and has left and right steering. So, the axial pressure and tangential bending loads will be applied to the main-branch of control arm, and the equivalent model is shown in Figure 11. Therefore, the bionic prototypes bearing both the axial pressure and tangential bending loads are a prerequisite for selection. 


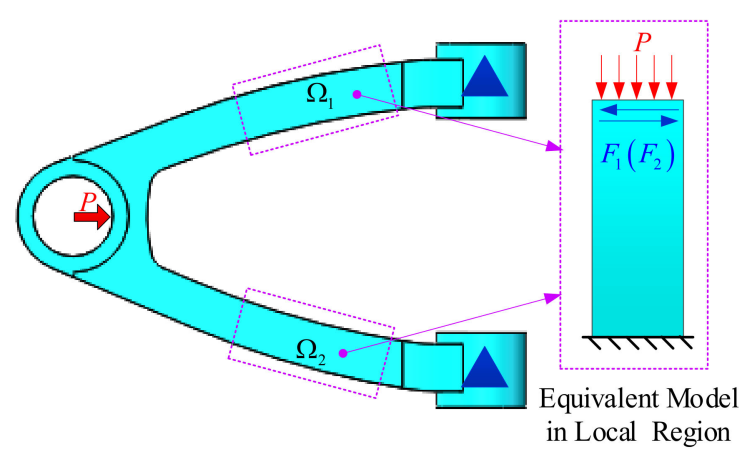

Figure 11. The load equivalent diagram on one side main-branch.

Based on the load similarity principle on the local area of bionic main-branch, it can be easily imagined that the behavior of fish is very similar to the load-bearing type of the bionic main-branch. Therefore, this case chooses the cross-section of fish bone as the bionic sub-branch. It is well known that the fish bone consists of main bones and secondary bones, as shown in Figure 12. If the cross-section of fish bone is directly applied to the bionic sub-branch, the comprehensive mechanical properties will not be brought into play because of the suspension of the secondary bone. Based on the above considerations, we need to add a skin structure to the suspension ends on both sides of the fish bone.

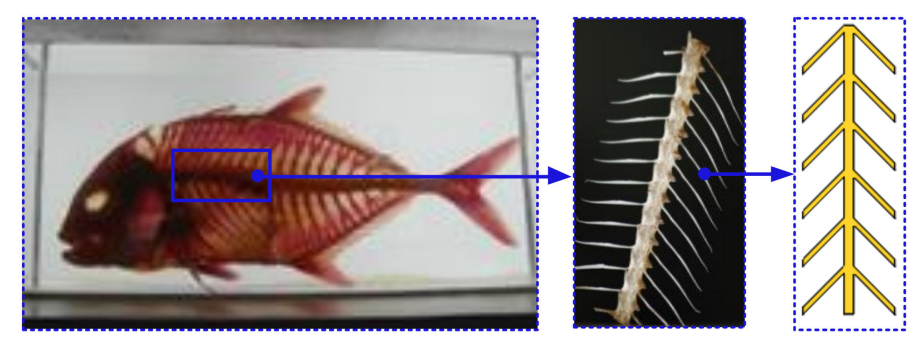

Figure 12. The schematic diagram of fishbone model.

The similarity analysis between the main-branch of control arm and the fish bone is as follows:

\subsubsection{Load Similarity}

When fish swim freely in the water, they will be resisted by frontal water flow and their heads will produce greater axial force. In addition, the swaying of the fish and the lateral impact of other organisms on the fish will cause the fish to be subjected to lateral force, which is similar to the equivalent load of the bionic main-branch of control arm.

\subsubsection{Structural Similarity}

Fish bone is composed of the main bone and the secondary bone, which is very similar to the design idea of main-branch and sub-branch in this paper. Its structure is a slender structure with high specific stiffness, and has good compressive and bending characteristics [39], which is similar to the main-branch of the control arm. In addition, the main bone and the secondary bone of the fish bone are connected at an appropriate angle, which has the characteristics of biological branching and can give full play to the material bearing performance.

\subsubsection{Manufacturability}

The main and secondary skeleton of fish bone correspond to the main-branch and sub-branch of the bionic structure respectively. By adding skins parallel to the main-branch at the suspended ends of the secondary bone, the bionic structure avoids the hollow structure commonly used in the skeleton 
structure, which can be accomplished by traditional manufacturing process. Therefore, it is feasible to select fish bone as bionic prototype, which is suitable for mass production and engineering application.

\subsection{Sub-Branch of the Control Arm}

After determining the bionic prototype, the bionic design of the sub-branch is carried out and the design idea is shown in Figure 13.

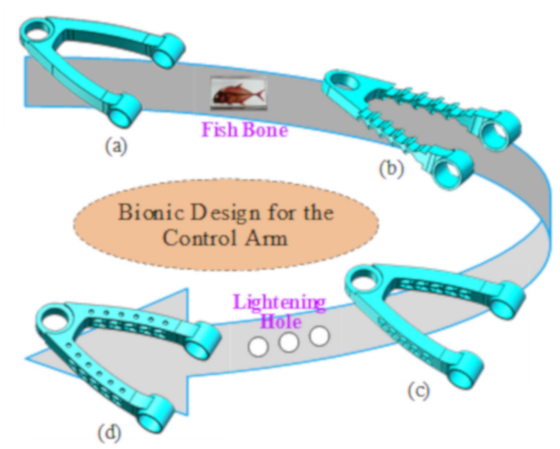

Figure 13. The design idea of bionic sub-branch: (a) the main-branch of control arm; (b) the bionic model with fish bone; (c) the bionic model with skin; (d) the final bionic model.

The fish bone structure is embedded in the bionic main-branch designed in Section 3.2 and the main bone of the fish bone is taken as the central axis of the main-branch and the secondary bone structure is added to both sides of the main bone, as shown in Figure 13b. It can be seen from Figure 12 that the angular of the main bone and the secondary bone is in the range of $30^{\circ} \sim 60^{\circ}, 45^{\circ}$ is selected as the initial degree and the optimal degree will be obtained by the size optimization in the next step. As the suspended secondary bone will not be able to exert the structural mechanical performance of fish bone, the skin structure is added at the suspension ends of the secondary bone, as shown in Figure 13c. Finally, the lightening round holes, which can be machined easily, are added to the upper and lower plates and the final bionic model is shown in Figure 13d.

\subsection{Multi-Objective Size Optimization}

Based on the above design, the parametric model of control arm is established and the multi-objective size optimization is carried out. This case takes the maximum structural stiffness and strength and the minimum mass as optimization objective, and several important dimensions as design variables are shown in Figure 14, including the diameter of lightening holes $\phi$, the angle of bionic stiffeners $\alpha$, the thickness of the control arm $h$, the thickness of upper, middle and lower plate $h_{1}, h_{2}, h_{3}$.

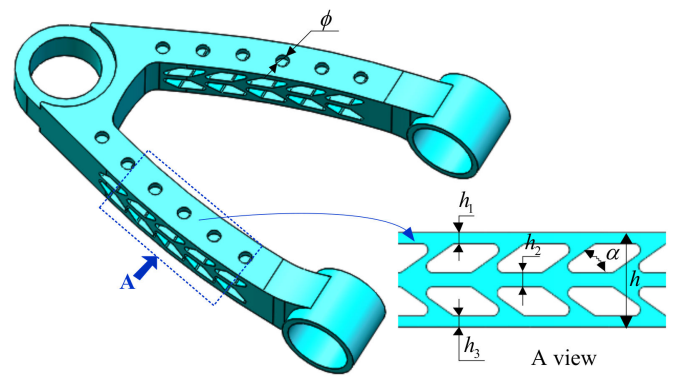

Figure 14. Parametric model of the control arm.

In order to improve the efficiency of size optimization, the sensitivity analysis is applied to obtain the influence degree of these dimensions to the optimization objective by the Equation (8) in Section 2.5, where, the $x_{i}$ is the vector of design variables $x_{i}=\left[h, \alpha, \phi, h_{1}, h_{2}, h_{3}\right]$. 
The parametric model in Figure 14 is input in the commercial FEA software ANSYS 18.0, and the sensitivity analysis is carried out. The result is shown in Figure 15. From the sensitivity analysis results, the bionic structure is mainly sensitive to the plate thickness and the angle of the secondary bone. Therefore, five parameters of $\alpha, h, h_{1}, h_{2}, h_{3}$ are selected as design variables.

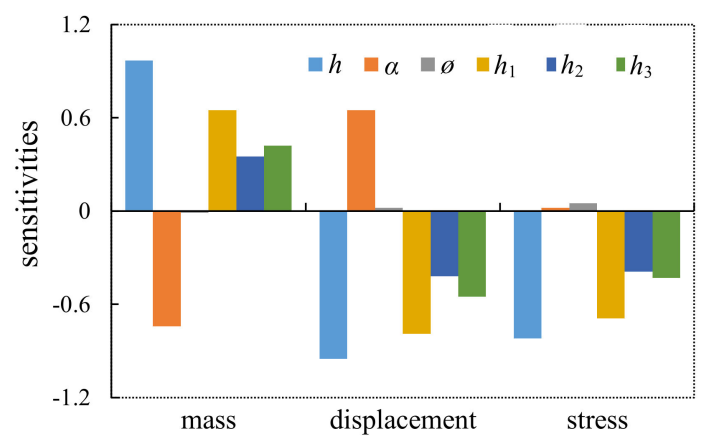

Figure 15. The sensitivity analysis.

According to the design and installation requirements of the control arm, if the size is too large, it will interfere with the control arm and other components and can not work normally. However, if the size is too small, it will not guarantee enough stiffness, and it will also make it difficult to apply biological characteristics to structural sub-branch. Therefore, the maximum and minimum values of the five design variables are set near the initial values, and the specific ranges are shown in Table 2.

Table 2. Variation range and initial value of design variables.

\begin{tabular}{cccc}
\hline Design Variables & Initial Value & Minimum Value & Maximum Value \\
\hline$h(\mathrm{~mm})$ & 32.0 & 25 & 35 \\
$h_{1}(\mathrm{~mm})$ & 3.5 & 3 & 5 \\
$h_{2}(\mathrm{~mm})$ & 4.5 & 3 & 5 \\
$h_{3}(\mathrm{~mm})$ & 3.5 & 3 & 5 \\
$\alpha\left({ }^{\circ}\right)$ & 45.0 & 40 & 50 \\
\hline
\end{tabular}

And the mathematical model of size optimization is established, as shown in Equation (10).

$$
\left\{\begin{array}{l}
\text { find } h, h_{1}, h_{2}, h_{3}, \alpha \\
\min f(x)=\min \left[M, d_{1}, \sigma_{1}, d_{2}, \sigma_{2}\right] \\
\text { s.t. } h \in[25,35] ; h_{1} \in[3,5] ; h_{2} \in[3,5] \\
\quad h_{3} \in[3,5] ; \alpha \in[40,50]
\end{array}\right.
$$

The FEM of the bionic model of the control arm is established and the size optimization is carried out. A set of optimum sizes are obtained and rounded, as shown in Table 3, the final bionic model is established.

Table 3. The results of size optimization.

\begin{tabular}{cccc}
\hline Parameters & Initial Value & Optimal Value & Round Value \\
\hline$h(\mathrm{~mm})$ & 32.0 & 29.91 & 30.0 \\
$h_{1}(\mathrm{~mm})$ & 3.5 & 3.86 & 4.0 \\
$h_{2}(\mathrm{~mm})$ & 4.5 & 4.11 & 4.0 \\
$h_{3}(\mathrm{~mm})$ & 3.5 & 3.93 & 4.0 \\
$\alpha\left({ }^{\circ}\right)$ & 45.0 & 42.80 & 43.0 \\
\hline
\end{tabular}


In order to verify the manufacturability, the control arms are machined by numerical control machine tool, as shown in Figure 16, the total weight of the control arm is reduced from $1.01 \mathrm{~kg}$ to $0.76 \mathrm{~kg}$.

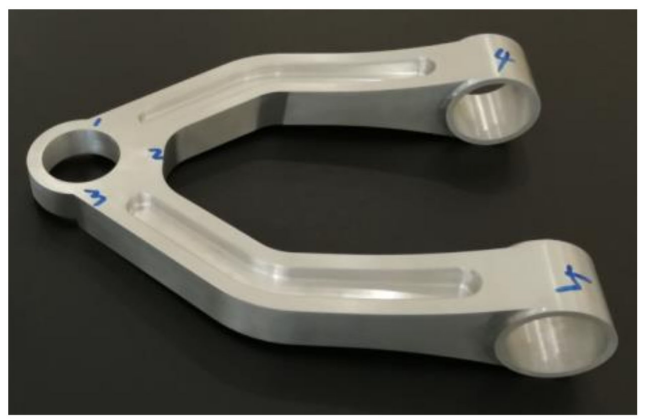

(a)

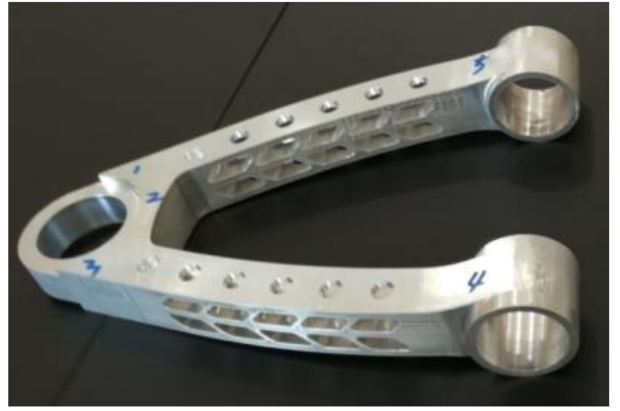

(b)

Figure 16. The samples of the control arm: (a) initial model $(1.01 \mathrm{~kg})$; (b) bionic model $(0.76 \mathrm{~kg})$.

\section{Results and Discussion}

The FEM of both the initial model and the bionic model are established in commercial FEA software ANSYS 18.0. The material properties shown in Table 1 are assigned, and the loads and constraints shown in Figure 8 are applied to two model.

Because the bionic model is more complex than the initial model, the meshing convergence is verified firstly by calculating the maximum displacement and stress with different element sizes. The variation trends under 1 4.5 mm element size are shown in Figure 17. From Figure 17a, the maximum displacements of the two models are constant under different element size, the value of initial model is $0.0611 \mathrm{~mm}$, while the value of bionic model is $0.0230 \mathrm{~mm}$. However, the maximum stress varies with the element size. The smaller the element size, the greater the local stress value in Figure 17b. The main reason is that too small element size is easy to cause stress concentration. However, the larger the element size, the lower the calculation accuracy. So, the convergence solutions of the two models can be obtained when the element size is $3 \mathrm{~mm}$, as shown in Figure 18.

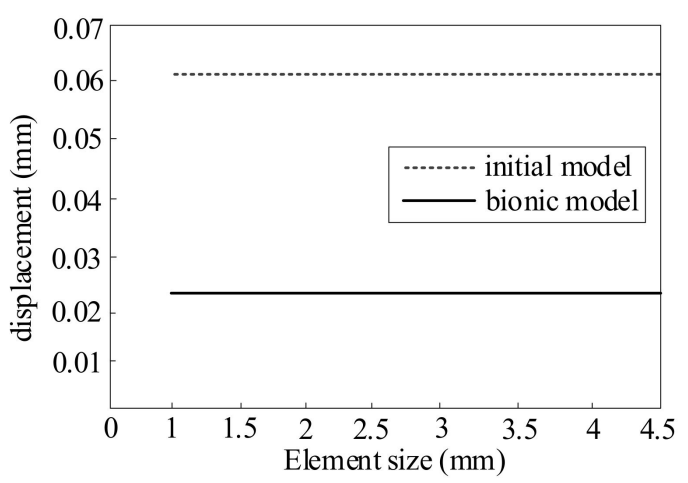

(a)

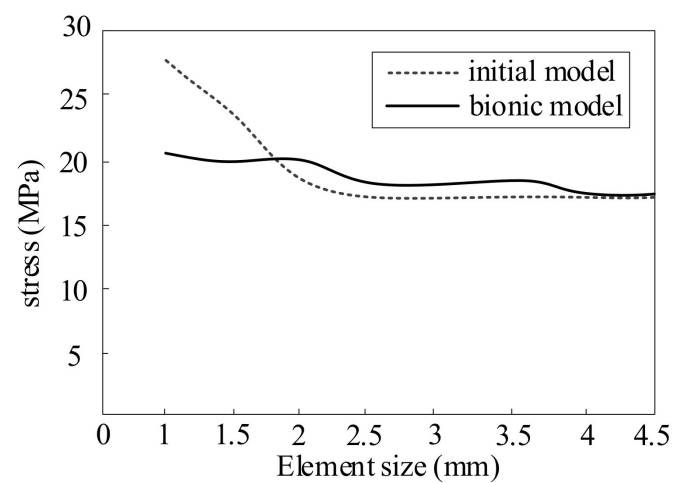

(b)

Figure 17. Verification of meshing convergence: (a) maximum displacement; (b) maximum stress. 


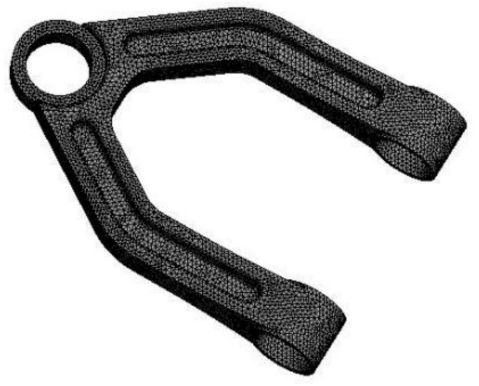

(a)

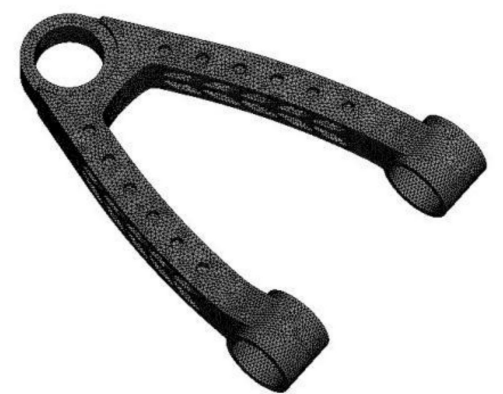

(b)

Figure 18. The finite element model (FEM) of the initial model and bionic model: (a) initial model; (b) bionic model.

Then, the FEA is carried out. The displacement and stress distribution are shown in Figures 19 and 20, and the comparisons of mechanical performance are shown in Table 4. It can be seen that the maximum displacement $(0.023 \mathrm{~mm})$ of bionic model is significantly reduced compared with that of the initial model $(0.061 \mathrm{~mm})$. The maximum stress $(18.69 \mathrm{MPa})$ is far less than the material yield strength (220 MPa). Although the maximum stress of the bionic model is slightly larger than that of the original model, but the value is only one tenth of the yield strength of the material, that is, the safety factor of the bionic model is 11.77 , which shows that the structure is absolutely safe. In addition, it is difficult to ensure the same stress when the structure is lighter. Under the design criteria, it is more reasonable to deteriorate the whole stress and greatly improve the structural stiffness and weight. Besides, it can be seen that the stress distribution of the bionic model is more uniform from Figures 19b and 20b.
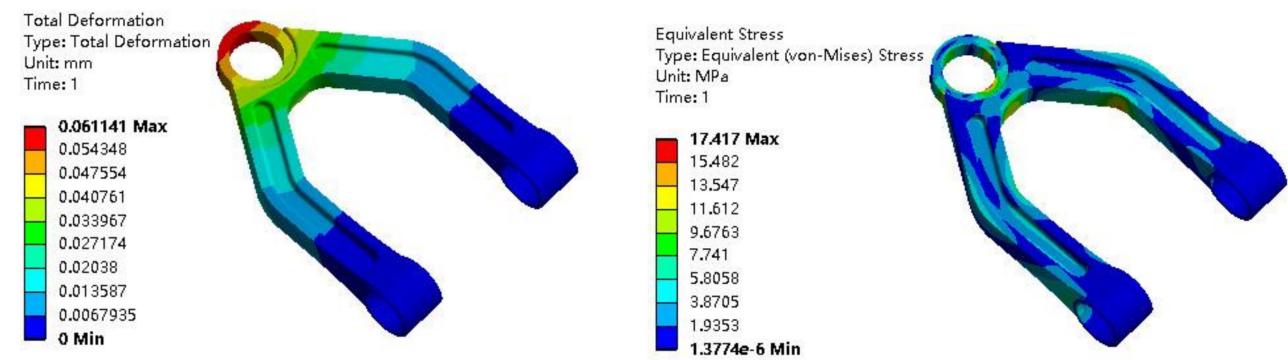

(a)

(b)

Figure 19. The finite element analysis (FEA) results of the initial model: (a) displacement distribution; (b) stress distribution.

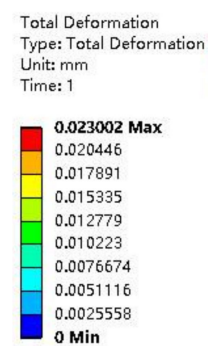

0.00255

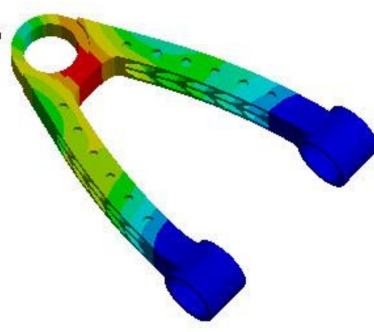

(a)

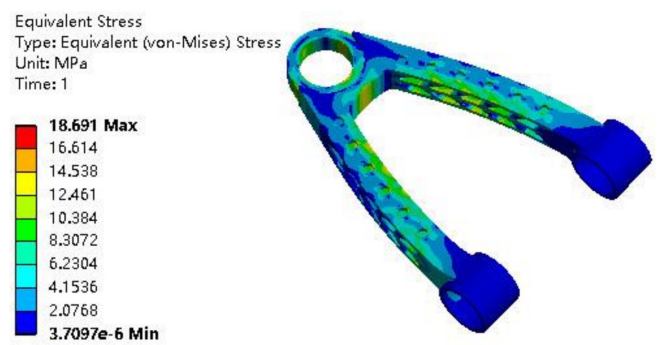

(b)

Figure 20. The FEA results of the bionic model: (a) displacement distribution; (b) stress distribution. 
Table 4. Mechanical performances of the new control arm.

\begin{tabular}{cccc}
\hline & Max Displacement $(\mathbf{m m})$ & Max Stress $(\mathbf{M P a})$ & Mass $(\mathbf{k g})$ \\
\hline Initial model & 0.061 & 17.42 & 1.01 \\
Bionic model & 0.023 & 18.69 & 0.76 \\
Variation & $-62.3 \%$ & $+7.3 \%$ & $-24.75 \%$ \\
\hline
\end{tabular}

As the strength of the control arm is far less than the material yield strength, it is not considered the main factor, while the stiffness directly affects the stability and sensitivity of the vehicle during driving. Therefore, the high stiffness design of the control arm is the focus in this case, and more materials should be distributed in the area of large load and deformation, rather than the area of stress concentration. So, we can conclude that the material distribution of the bionic model is more reasonable than the initial model. The stiffness is increased by $62.3 \%$ while the weight is reduced by $24.75 \%$.

In this study, the new control arm is tested on a real vehicle to further verify the mechanical performances. The sample is installed in a new formula car for vehicle testing, including two extreme conditions: wheel side impact on the obstacle, vehicle side slip and brake, as illustrated in Figure 21. The test results show that the control arm can meet the requirements of stiffness, strength and sensitivity in a normal driving process.

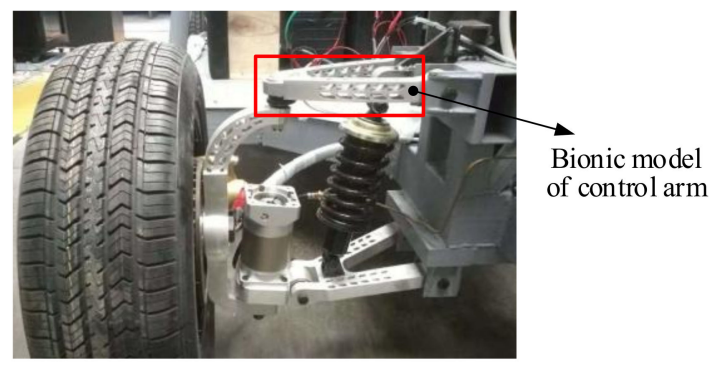

Figure 21. Real vehicle test of the new control arm.

\section{Conclusions}

A novel bionic design method for the skeleton structures is proposed by the bio-inspired idea of main-branch and sub-branch. The main-branch of the bionic structure is obtained by the load path analysis, which can get a clear load transfer skeleton. The loads on a given structure are equivalent to the main-branch of the bionic structure and the bionic prototype is selected from three aspects: load similarity, structural similarity and manufacturability. The efficiency of selecting a bionic prototype is improved by the local similarity of the main-branch instead of the whole similarity. Then, the sub-branch of the bionic structure is established based on the cross-sectional characteristics of a bionic prototype, and the size optimization provides detailed size value for each parameter that achieve secondary lightweight.

Taking a control arm of automobile suspension system as a case, the results of bionic design show that the material distribution of the bionic model is more reasonable than the initial model, and structural stiffness is increased by $62.3 \%$, while the weight is reduced by $24.75 \%$. This method combines the load path theory with biological feature, which can realize the lightweight design of more complex structure compared with the traditional bionic method.

In addition, the selection of bionic prototype mainly focuses on load similarity and then the structural similarity, which can expand the selection range of biological prototype. The manufacturability is considered firstly in bionic design. Although the selected bionic prototype is still subject to certain constraints in this study, in the future, the selection range of bionic prototype will be further expanded under the development of additive manufacturing. For example, the cross-section characteristics of bamboo, wheat straw and bone will also be used for the main-branch of the control 
arm. Therefore, this method can also be used for the skeleton structures of other fields including automobile, aerospace and civil engineering.

Author Contributions: Bionic design method, Z.W. and F.W.; the theory of load path analysis, Z.W., N.W. and Q.W.; bionic design of the control arm, Z.W., Y.L. and Q.Y.; numerical simulation, Z.W. and Q.Y.; validation, Z.W. and F.W.; writing, review and editing, Z.W., Q.W. and N.W. All authors have read and agreed to the published version of the manuscript.

Funding: This research was supported by the Chinese National Key Research and Development Program (2016YFC0802900), the Key Natural Science Projects of Hebei Provincial Department of Education (ZD2020156), the Science and Technology Research Project of Colleges and Universities in Hebei Province (Grant NO. QN2018228).

Conflicts of Interest: The authors declare no conflict of interest.

\section{References}

1. Qu, Y.; Jiang, Y.; Feng, L.; Li, X.; Liu, B.; Wang, W. Lightweight design of multi-objective topology for a large-aperture space mirror. Appl. Sci. 2018, 8, 2259. [CrossRef]

2. Ghasemi, S.; Zohrevand, P.; Mirmiran, A.; Xiao, Y.; Mackie, K. Super lightweight UHPC-HSS deck panel for movable bridges. Eng. Struct. 2016, 113, 186-193. [CrossRef]

3. Zhang, T.; Wang, A.; Wang, Q.; Guan, F. Bending characteristics analysis and lightweight design of a bionic beam inspired by bamboo structures. Thin Walled Struct. 2019, 142, 476-498. [CrossRef]

4. Dong, Y.; Zhu, G. Mechanical analysis and bionic structure design of astronautic payloads based on natural honeycomb. J. Astronaut. 2016, 37, 262-267.

5. Drack, M.; Limpinsel, M.; De, G.; Nebelsick, J.; Betz, O. Towards a theoretical clarification of biomimetics using conceptual tools from engineering design. Bioinspir. Biomim. 2017, 13, 016007. [CrossRef]

6. Ding, Y.; Zhou, Z.; Wang, Z.; Liu, H.; Wang, K. Bionic stiffener layout optimization with a flexible plate in solar-powered uav surface structure design. Appl. Sci. 2019, 9, 5196. [CrossRef]

7. Xie, Y.; Bai, H.; Liu, Z.; Chen, N. A Novel Bionic Structure Inspired by Luffa Sponge and Its Cushion Properties. Appl. Sci. 2020, 10, 2584. [CrossRef]

8. Wang, C.; Li, Y.; Zhao, W.; Zou, S.; Zhou, G.; Wang, Y. Structure design and multi-objective optimization of a novel crash box based on biomimetic structure. Int. J. Mech. Sci. 2018, 138, 489-501. [CrossRef]

9. Zhao, L.; Ma, J.; Chen, W.; Guo, H. Lightweight design and verification of gantry machining center crossbeam based on structural bionics. J. Bionic Eng. 2011, 8, 201-206. [CrossRef]

10. Kim, D.; Jung, S.; Lee, J.; Chang, S. Parametric study on design of composite-foam-resin concrete sandwich structures for precision machine tool structures. Compos. Struct. 2006, 75, 408-414. [CrossRef]

11. Kakoty, N.; Hazarika, S. A biomimetic similarity index for prosthetic hands. In Proceedings of the IEEE Symposium on Computational Intelligence in Rehabilitation and Assistive Technologies, Singapore, 16-19 April 2013; pp. 32-39.

12. Gauchia, A.; Diaz, V.; Boada, M.; Boada, B. Torsional stiffness and weight optimization of a real bus structure. Int. J. Automot. Technol. 2010, 11, 41-47. [CrossRef]

13. Zhang, H.; Ding, X.; Dong, X.; Xiong, M. Optimal topology design of internal stiffeners for machine pedestal structures using biological branching phenomena. Struct. Multidiscip. Optim. 2017, 57, 2323-2338. [CrossRef]

14. Li, B.; Hong, J.; Liu, Z. Stiffness design of machine tool structures by a biologically inspired topology optimization method. Int. J. Mach. Tools Manuf. 2014, 84, 33-44. [CrossRef]

15. $\mathrm{Li}, \mathrm{Y} . ; \mathrm{Xue}, \mathrm{K}$. Mechanics in leaf venation morphogenesis and their biomimetic inspiration to construct a 2-dimensional reinforcement layout model. J. Biomim. Biomater. Tissue Eng. 2011, 10, 81-93. [CrossRef]

16. Guo, S.; Yuan, Z.; Wu, F.; Li, Y.; Wang, S.; Qin, S.; Peng, Q. TRIZ application in bionic modeling for lightweight design of machine tool column. In Proceedings of the International Design Engineering Technical Conferences and Computers and Information in Engineering Conference, Quebec City, QC, Canada, 26-29 August 2018; p. 85516.

17. Liu, X.; Huang, S.; Chen, Y. Research and application: Conceptual integrated model based on TRIZ and bionics for product innovation. Int. J. Interact. Des. Manuf. 2016, 11, 341-349.

18. Mann, D. Systematic technology transfer from biology to engineering. Philos. Trans. R. Soc. A 2002, 360, 159-173.

19. Emmelmann, C.; Sander, P.; Kranz, J.; Wycisk, E. Laser additive manufacturing and bionics: Redefining lightweight design. Phys. Procedia 2011, 12, 364-368. [CrossRef] 
20. Maier, M.; Siegel, D.; Thoben, K.; Niebuhr, N.; Hamm, C. Transfer of natural micro structures to bionic lightweight design proposals. J. Bionic Eng. 2013, 10, 469-478. [CrossRef]

21. Smarslik, M.; Ahrens, M.; Mark, P. Toward holistic tension -or compression-biased structural designs using topology optimization. Eng. Struct. 2019, 199, 109632. [CrossRef]

22. Guirguis, D.; Hamza, K.; Aly, M.; Hegazi, H.; Saitou, K. Multi-objective topology optimization of multi-component continuum structures via a Kriging-interpolated level set approach. Struct. Multidiscip. Optim. 2014, 51, 733-748. [CrossRef]

23. Wächter, M.; Leicher, M.; Hupka, M.; Leistner, C.; Masendorf, L.; Treutler, K.; Kamper, S.; Esderts, A.; Wesling, V.; Hartmann, S. Monotonic and fatigue properties of steel material manufactured by wire arc additive manufacturing. Appl. Sci. 2020, 10, 5238. [CrossRef]

24. Li, Y.; Harrysson, O.; West, H.; Cormier, D. Mechanical properties of 3D re-entrant honeycomb auxetic structures realized via additive manufacturing. Int. J. Solids Struct. 2015, 69-70, 475-490.

25. Tamijani, A.; Gharibi, K.; Kobayashi, M.; Kolonay, M. Load paths visualization in plane elasticity using load function method. Int. J. Solids Struct. 2018, 135, 99-109. [CrossRef]

26. Wang, Q.; Zhang, G.; Sun, C.; Wu, N. High efficient load paths analysis with U* index generated by deep learning. Comput. Method Appl. Mech. Eng. 2019, 344, 499-511. [CrossRef]

27. Pejhan, K.; Kuznetcov, A.; Wang, Q.; Wu, Q.; Telichev, I. Design assessment of a multiple passenger vehicle component using load transfer index $\left(U^{*}\right)$ method. Int. J. Mech. Mater. Des. 2017, 14, 213-229. [CrossRef]

28. Kelly, D.; Reidsema, C.; Lee, M. An algorithm for defining load paths and a load bearing topology in finite element analysis. Eng. Comput. 2011, 28, 196-214. [CrossRef]

29. Takahashi, K.; Omiya, M.; Iso, T.; Zaiki, Y.; Sakurai, T.; Maki, T.; Urushiyama, Y.; Naito, T. Load transfer ustar $\left(U^{*}\right)$ calculation in structures under dynamic loading. Trans. Jpn. Soc. Mech. Eng. Part A 2013, 79, 1657-1668. [CrossRef]

30. Wang, E.; Nohara, T.; Ishii, H.; Hoshino, H.; Takashi, K. Load transfer analysis using indexes $U^{*}$ and $U^{* *}$ for truck cab structures in initial phase of frontal collision. Adv. Mater. Res. 2011, 156-157, 1129-1140. [CrossRef]

31. Wang, Q.; Zhou, W.; Telichev, I.; Wu, Q. Load transfer analysis of a bus bay section under standard rollover test using U*M index. Int. J. Automot. Technol. 2018, 19, 705-716. [CrossRef]

32. Wang, Q.; Pejhan, K.; Telichev, I.; Wu, Q. Demonstration of the effectiveness of $\mathrm{U}^{*}$-based design criteria on vehicle structural design. Proc. Inst. Mech. Eng. Part D J. Automob. Eng. 2018, 232, 995-1002. [CrossRef]

33. Takahashi, K. Relative rigidity of structures and Saint Venant's principle. Trans. Jpn. Soc. Mech. Eng. Ser. A 1986, 52, 2615-2621. [CrossRef]

34. Boudaoud, A. An introduction to the mechanics of morphogenesis for plant biologists. Trends. Plant Sci. 2010, 15, 353-360. [CrossRef] [PubMed]

35. Cai, K.; Shi, J. A bionic approach for topology optimization for tension-only or compression-only design. J. Bionic Eng. 2010, 7, 397-404. [CrossRef]

36. Waldron, D.; Harwood, J. A study of the relationship between bending rigidity and the ease of decortication of flax straw. J. Nat. Fibers 2010, 7, 42-60. [CrossRef]

37. Xu, H.; Li, W.; Li, M.; Hu, C.; Zhang, S.; Wang, X. Multidisciplinary robust design optimization based on time-varying sensitivity analysis. J. Mech. Sci. Technol. 2018, 32, 1195-1207. [CrossRef]

38. Ashtekar, J.; Thakur, A. Simulink model of suspension system and it's validation on suspension test rig. Int. J. Mech. Eng. Robot. Res. 2014, 3, 811-818.

39. Rivero, A.; Weaver, P.; Cooper, J.; Woods, B. Parametric structural modelling of fish bone active camber morphing aerofoils. J. Intell. Mater. Syst. Struct. 2018, 29, 2008-2026. [CrossRef]

Publisher's Note: MDPI stays neutral with regard to jurisdictional claims in published maps and institutional affiliations. 